ARTIGO ORIGINAL ORIGINAL ARTICLE

\section{Análise da produtividade da política de fusão de unidades hospitalares em Portugal integradas no Serviço Nacional de Saúde}

\author{
Productivity analysis of hospital mergers in \\ Portuguese National Health System \\ Alexandre Morais Nunes ${ }^{1}$ \\ DOI: 10.21115/JBES.v9.n1.p93-9
}

\begin{abstract}
RESUMO
Objetivo: Avaliar a variação da produtividade dos hospitais sujeitos a uma política de fusão entre os anos 2005 e 2013. Métodos: Para a medição da produtividade recorreu-se ao índice de Malmquist, que considera em simultâneo a variação da eficiência e a variação da tecnologia (fronteira ou melhores práticas). A população-alvo foram os Centros Hospitalares criados entre 2003 e 2010. Os dados de custos e da produção realizada foram obtidos através da revisão crítica da literatura (relatórios de gestão do Ministério da Saúde). Foram comparados os Centros Hospitalares criados antes e depois da fusão com os hospitais que não foram submetidos a este processo. Resultados: $60 \%$ dos hospitais não apresentaram melhoria de produtividade com a reforma em estudo. A produtividade média antes da fusão era de 1,004 e após fusão desceu para 0,977. Os hospitais não sujeitos a processo de fusão apresentaram melhores resultados com produtividade média de 0,994. Conclusões: A política de fusão de unidades de saúde não gerou ganhos de produtividade no médio prazo e os resultados em média demonstraram-se menos positivos no período pós fusão.
\end{abstract}

\section{Keywords:}

efficiency, productivity, cost and analysis cost, production, hospital mergers

\begin{abstract}
Objective: Evaluation of the productivity in hospitals merger between 2005 and 2013. Methods: Malmquist index for the measurement of productivity resorted to the Malmquist index. This method considers the simultaneous variation of the efficiency and the variation of technology (best practices). The target population were the hospitals created between 2003 and 2010. Data were obtained from critical literature review (Ministry of Health management reports). The model considers the change in efficiency and the change in technology, achieved on the basis of cost and production data held for the hospital centers created before and after the merger and for comparison with the hospitals that were not merged. Results: $60 \%$ of hospitals showed no improvement in productivity with the reform in the study, the average productivity before the merger was 1,004 and after melting down to 0.977. Hospitals not subject to merger process showed better results with an average yield of 0.994. Conclusions: The health units merger policy does not generate productivity gains in the medium term and the results showed on average are less positive.
\end{abstract}

Recebido em: 19/10/2016. Aprovado para publicação em: 23/03/2017.

1. Doutor em Administração da Saúde pela Universidade de Lisboa.

Financiamento: Não houve recebimentos de financiamento ou equipamento.

Autor correspondente: Alexandre Morais Nunes. Telefone cellular: 00351 967435705. E-mails: alexandre.nunes@ms.gov.pt / alexandre.m.nunes@tecnico.ulisboa.pt 


\section{Introdução}

A reforma da gestão hospitalar em Portugal teve inicio em 2002 com a adoção de práticas empresariais pelos hospitais públicos (Nunes, 2016). Na base deste processo estava a necessidade de garantir mais eficiência, menos custos, melhor qualidade, maior satisfação e mais acesso aos cuidados de saúde (Campos, 2007,2008).

Apesar destes objetivos bem claros, dois anos depois, o crescimento com as despesas públicas em saúde continuava a crescer e estava em risco a sustentabilidade do setor público em Portugal (Harfouche, 2012). Só os hospitais a essa data eram responsáveis por uma despesa superior a 50\% do total de despesas do Serviço Nacional de Saúde (Barros, 2013). Ao mesmo tempo crescia a procura por hospitais privados e também a despesa dos hospitais públicos com o setor convencionado (protocolos com prestadores privados) (Fernandes \& Nunes, 2016).

Como os principais problemas foram a ineficiência das práticas hospitalares de gestão, havia a necessidade de levar os gestores públicos a gerenciar melhor os recursos disponíveis (Barros, 2013). Dessa forma, em 2005, o governo decidiu que deveria melhorar os resultados, capacitando os gestores com práticas de gestão empresarial (Campos, 2008). Na verdade, este momento foi criticado por alguns gestores pois em tão pouco tempo se transitou de práticas de gestão puramente públicas (até 2002), para um modelo de Sociedade Anónima (empresarial) (entre 2002 e 2004) e para um modelo de Empresa Pública do Estado muito próximo do empresarial (a partir de 2005) (Nunes \& Harfouche, 2015).

No mesmo ano, e para assegurar a sustentabilidade do sistema público de saúde, o Governo fez uma nova aposta com base na fusão de hospitais que tinham diferentes dimensões, capacidades e especificidades. Estas novas unidades ficaram conhecidas como Centro Hospitalares. De acordo com Correia de Campos (2008), Ministro da Saúde responsável pelas reformas, a luta pela eficiência e aumento de produtividade dos hospitais com gestão empresarial, não seria possível sem uma forte concentração de unidades com estatuto juridico de entidades públicas empresariais (EPE) (Campos, 2008, p.42).

O principal objetivo da política de fusão de hospitais era a promoção da utilização de todos os recursos e potencialidades existentes nos hospitais do SNS que estavam muitas vezes sub-utilizados (como equipamentos e meios tecnológicos) e promover um maior investimento no acesso à inovação e na formação de recursos humanos (Campos, 2008).

O legislador defendia na publicação da redação legal que criava os Centros Hospitalares:

- A interconexão dos serviços e hospitais na mesma região e que desempenham as mesmas funções;

- A melhor utilização da capacidade existente em cada hospital público;
- A concentração das especialidades médicas e da inovação tecnológica dentro da mesma equipa de gestão;

- Alcançar ganhos de eficiência;

- A maior produtividade das unidades como um todo (Decreto-Lei n. ${ }^{\circ}$ 284/99, de 26 de julho).

Em defesa desta política de fusão, conhecida como "hospital mergers" na literatura internacional, é defendida a teoria da perspetiva de crescimento em função de economias de escala, tendo por base que algumas das quais ainda estavam por explorar (Azevedo \& Mateus, 2013; Dafny, 2009; Tenn, 2011). Como exemplos de contribuição para as economias de escala, na literatura internacional, podemos encontrar referências à distribuição das despesas fixas dos hospitais separados por um único e maior volume produtivo; à diminuição de custos de administração; ao melhor aproveitamento de recursos com nova afetação dos recursos subutilizados e ainda algumas melhorias na qualidade e no acesso, já que os meios tecnológicos e exames complementares estariam centralizados num só local, dispondo o doente de acesso à mais alta tecnologia e com inovação crescente (Given, 1997; Sinay, 1998; Tenn, 2011).

Em relação ao caso português, foi reconhecido por Barros (2013), que existiam unidades com economias de escala por implementar e outras já sobre dimensionadas. No entanto, a implementação da fusão de hospitais em Portugal tem seguido as recomendações internacionais de vários autores que estudaram exemplos ocorridos. Estas referências internacionais referiam que as as economias de escala apenas se justificariam se aplicadas ao processo de reforma que juntariam hospitais de pequena (menos de 200 camas) com hospitais de maiores dimensões (Brooks \& Jones, 1997; Given, 1996; Ferrier \& Valdmanis, 2004; Sinay, 1998). Caso contrário, ao fundir hospitais pequenos com outros mais pequenos ou hospitais grandes com outros maiores incorria-se no risco de não se gerar economias de escala no primeiro caso e no segundo gerar mesmo uma maior subutilização (efeito contrário ao pretendido) (Sinay, 1998).

Em Portugal, a fusão de unidades de saúde assumiu uma perspectiva de integração horizontal, além da criação de Centros Hospitalares (perspectiva de integração vertical). Este outro tipo de fusão, resultava da junção de unidades de atenção primária com hospitais que ficariam sobre a mesma administração numa área geográfica próxima, gerando o que ficou conhecido na literatura da gestão hospitalar como Unidades Locais de Saúde (Campos \& Simões, 2011; Santana \& Costa, 2008).

Neste artigo efetua-se uma análise da evolução da produtividade registrada com a fusão de hospitais, que se desenvolveu desde o ano 2005 sob o estatuto de Empresa Pública do Estado. A avaliação desta medida ganha relevo na literatura internacional, acrescentado valor pois a maioria 
dos estudos referem-se a hospitais norte americanos e são poucos os que analisam as diferenças de produtividade antes e após a fusão dos hospitais.

\section{Métodos}

Foi aplicado o modelo de apuramento da produtividade, conhecido como índice de produtividade de Malmquist. Este é um índice bilateral que, com base no apuramento da eficiência computacional, permite comparar a tecnologia de produção de duas economias (Caves, Christensen \& Diewert, 1982).

Este modelo de análise da produtividade, proposto pela primeira vez em 1982 por Caves, Christensen e Diewert (1982), obedece a uma sequência operacional que inclui a variação simultânea da eficiência e das melhores práticas (variação da fronteira da tecnologia) ao longo de vários períodos (Lovell, 2006). A seleção deste índice teve em conta a sua capacidade de ser utilizado em casos de tecnologias de produção que apresentam vários inputs (entradas de recursos) e outputs (saídas/produção), pelo facto de não necessitar de dados relativos aos preços de cada recurso utilizado nem de cada produto final produzido e ainda pela possibilidade de obtenção de informação sobre as razões que estão por trás das variações observadas da produtividade (Vasquez, 2012).

A operacionalização deste índice é efetuada através de um cálculo que resulta do produto da variação da eficiência ("catch-up") (VE) pela variação da tecnologia (fronteira, inovação ou melhores práticas) (VT) (Cooper et al., 2007; Vasquez, 2012).

\section{$\mathrm{IM}=V E \mathrm{x} V T$}

Sendo,

$$
V E=\frac{\delta_{t+k}^{\mathrm{t}+\mathrm{k}}}{\delta_{t}^{\mathrm{t}}} \quad \text { e } \quad V T=\sqrt{\frac{\delta_{\mathrm{t}}^{\mathrm{t}}}{\delta_{t}^{\mathrm{t}+\mathrm{k}}} \times \frac{\delta_{\mathrm{t}+\mathrm{k}}^{\mathrm{t}}}{\delta_{\mathrm{t}+\mathrm{k}}^{\mathrm{t+k}}}}
$$

e,

$\delta_{t}^{t} \quad$ Orácio de eficiência relativa no período " $\mathrm{t}$ " medida pela fronteira do período " $\mathrm{t}$ "

$\delta_{t}^{t+k}$ O rácio de eficiência relativa no período " $\mathrm{t}$ " medida pela fronteira do período " $\mathrm{t}+\mathrm{k}$ "

$\delta_{\mathrm{t}+\mathrm{k}}^{\mathrm{t} \mathrm{k}} \mathrm{O}$ rácio de eficiência relativa no período " $\mathrm{t}+\mathrm{k}$ " medida pela fronteira do período " $t+k$ "

$\delta_{\mathrm{t}+\mathrm{k}}^{\mathrm{t}} \mathrm{O}$ rácio de eficiência relativa no período " $\mathrm{t}+\mathrm{k}$ " medida pela fronteira do período " $\mathrm{t}$ "

Assim, resolvendo a equação se obtém uma expressão mais simplificada para o apuramento do índice de Malmquist:

$$
\mathrm{IM}=\sqrt{\frac{\delta_{\mathrm{t}+\mathrm{k}}^{\mathrm{t}}}{\delta_{t}^{\mathrm{t}}} \times \frac{\delta_{\mathrm{t}+\mathrm{k}}^{\mathrm{t}}}{\delta_{t}^{\mathrm{t}+\mathrm{k}}}}
$$

As variáveis selecionadas como inputs do modelo foram os custos dos hospitais. Os custos totais dos hospitais compreendem todas as demonstrações de contas (custos com pessoal, com produtos e bens de consumo, custo com medicamentos e outros custos indiretos), exceto amortizações, custos com perdas financeira e custos extraordinários. Esta variável é expressa a preços constantes de 2003 operacionalizada pelo deflator do Produto Interno Bruto. As variáveis clinicas de avaliação da produtividade, os outputs, foram: o número de episódios de emergências, o número de doentes internados, o número de cirurgias e o número de consultas nos hospitais. A população alvo foram os vinte Centros Hospitalares, envolveram 44 hospitais, com a aplicação da política de junção entre os anos 2003 e 2013.

A estratégia de seleção de um período mais reduzido (até 2012) tem por justificação o fato de se pretender uma avaliação rigorosa com dados validados e com uma visão de médio prazo. Daí serem contempladas as reformas com num período máximo de 10 anos e mínimo de 3 anos.

A análise dos resultados obtidos fez-se em duas etapas. Numa primeira etapa analisou-se, através de uma perspectiva global a evolução da produtividade para as unidades sujeitas a esta política antes e depois da fusão. Numa segunda etapa estabeleceu-se uma comparação com a evolução da produtividade nas unidades não sujeitas a esta política. Assim:

- Para a primeira etapa foram selecionados 20 centros hospitalares constituídos por 44 unidades hospitalares. De forma a apurar a produtividade das unidades, antes do período de fusão, foram considerados os somatórios dos inputs e outputs dos hospitais que vieram a constituir os $\mathrm{CH}$ em análise. Apenas desta forma seria possível comparar os efeitos da política de concentração de unidades de saúde através deste índice;

- Para a segunda etapa foi avaliada a produtividade de cinco hospitais, não sujeitos a esta política de concentração, para estabelecer uma comparação com os restantes. Os hospitais selecionados foram: o Hospital Garcia da Horta; o Hospital Distrital de Santarém; o Hospital Distrital Santa Maria Maior, o Hospital Distrital da Figueira da Foz e o Hospital do Espirito Santo.

Não foram contempladas no modelo outras reformas como a fusão de unidades hospitalares com unidades de atenção primária pois não têm igual produção nem os mesmos custos. Os dados incluídos foram obtidos de revisão crítica da literatura (relatórios de gestão) e validados pelas entidades competentes do Ministério da Saúde.

\section{Resultados}

O impacto dos processos de fusão das unidades hospitalares foi calculado para cada ano com recurso ao modelo mate- 
mático proposto pelo Índice de Malmquist e foi realizado em duas etapas.

Os resultados referentes à evolução da produtividade dos Centros Hospitalares antes e após a fusão (etapa 1 do modelo) levou ao apuramento da produtividade dos centros hospitalares em cada ano, como se apresenta na tabela 1.

A tendência registrada é a de que ao longo do tempo a maioria dos centros hospitalares aumenta a sua produtividade, o que é um sinal positivo dos objetivos da política de saúde. No entanto quando observada a média global de produtividade do grupo dos Centros Hospitalares registram-se as maiores falhas no ano de 2010/2011, que corresponde a um ano de intensa crise econômica portuguesa com intervenção externa do Fundo Monetário Internacional.

Após este ano fatídico de crise é preciso ter cuidado ao afirmar a recuperação da produtividade no ano 2012. Isto porque é facto que a produtividade aumentou mas o aumento não se deveu a um melhor aproveitamento de recursos e consequentes poupanças associadas, mas sim à redução do acesso com o aumento dos pagamentos a cargo dos doentes (taxas moderadoras), com a redução de apoios sociais e de transporte (o que baixou o acesso) e com o corte do financiamento dos hospitais. Este ultimo ponto é importante para a metodologia aplicada, uma vez que os custos são o input do calculo da variação da eficiência e a redução de custos tem no imediato um efeito positivo na produtividade pois os cálculos não distinguem os cortes das poupanças eficientes de recursos, apenas considera a redução dos custos.

Mas, analisando caso a caso, é preciso ter em conta que as fusões de hospitais não decorreram todas ao mesmo tempo e uns centros hospitalares foram criados em 2005 e outros em 2008 (por exemplo). Por isso, se fez um apuramento da média de produtividade de cada hospital (caso a caso) antes de se transformar em Centro Hospitalar e nos anos que se seguiram à fusão (Tabela 2).

Para uma melhor observação das variações se apresenta na figura 1 a apresentação gráfica dos resultados.

Ao analisar os dados se verifica que os hospitais antes da junção apresentavam um crescimento anual positivo de 0,4\% e após a transformação em Centros Hospitalares a produtividade caiu $2,73 \%$. Com este resultado podemos concluir que a opção reformadora não teve os efeitos previstos.

No entanto, a quebra não foi geral pois houve hospitais que cresceram e apresentaram resultados positivos. Este caso aplicou-se a 8 Centros Hospitalares que após fusão beneficiaram de uma melhoria da sua produtividade. São

Tabela 1. Evolução da produtividade dos centros hospitalares, no período 2002-2012

\begin{tabular}{|c|c|c|c|c|c|c|c|c|c|c|}
\hline $\mathrm{CH}$ & $2003 / 2$ & $2004 / 3$ & $2005 / 4$ & $2006 / 5$ & $2007 / 6$ & $2008 / 7$ & $2009 / 8$ & $2010 / 9$ & $2011 / 10$ & $2012 / 11$ \\
\hline CH de São João & 0,964 & 0,969 & 0,858 & 1,102 & 0,977 & 0,960 & 1,018 & 1,002 & 0,955 & 1,064 \\
\hline $\mathrm{CH}$ Alto Ave & 1,006 & 1,017 & 1,231 & 1,231 & 1,116 & 1,007 & 0,794 & 1,291 & 0,515 & 1,559 \\
\hline CH Médio Ave & 1,249 & 0,984 & 1,009 & 1,016 & 1,005 & 1,000 & 1,004 & 0,691 & 0,916 & 1,384 \\
\hline $\mathrm{CH}$ entre Douro e Vouga & 1,019 & 0,990 & 0,958 & 0,940 & 0,962 & 1,006 & 1,006 & 1,000 & 0,861 & 1,107 \\
\hline CH Tâmega e Sousa & 1,128 & 1,073 & 0,998 & 0,956 & 1,048 & 1,099 & 0,969 & 0,979 & 1,013 & 0,982 \\
\hline CH Trás-os-Montes e Alto Douro & 0,988 & 0,953 & 0,994 & 0,991 & 1,198 & 1,159 & 0,795 & 1,419 & 0,569 & 1,421 \\
\hline $\mathrm{CH}$ do Nordeste & 1,004 & 0,916 & 0,971 & 1,016 & 0,946 & 1,079 & 0,998 & 1,003 & - & - \\
\hline $\mathrm{CH}$ do Porto & 1,023 & 0,976 & 0,986 & 1,013 & 0,976 & 0,824 & 0,834 & 0,999 & 1,003 & 0,983 \\
\hline CH V.N.Gaia/Espinho & 0,927 & 0,787 & 0,969 & 0,971 & 0,874 & 1,034 & 0,971 & 0,944 & 0,563 & 1,443 \\
\hline CH Leiria-Pombal & 1,050 & 1,226 & 0,923 & 1,523 & 1,018 & 1,021 & 1,281 & 1,021 & 0,912 & 1,423 \\
\hline $\mathrm{CH}$ Tondela-Viseu & 1,050 & 0,970 & 0,998 & 0,995 & 0,992 & 1,003 & 0,985 & 0,988 & 0,948 & 1,011 \\
\hline $\mathrm{CH}$ do Baixo Vouga & 1,115 & 0,955 & 0,884 & 1,069 & 0,929 & 1,131 & 0,974 & 0,936 & 0,696 & 1,117 \\
\hline CH Universitário de Coimbra & 1,035 & 0,944 & 1,101 & 0,983 & 1,005 & 0,982 & 0,978 & 0,998 & 0,811 & 1,497 \\
\hline CH Barreiro - Montijo & 1,003 & 0,987 & 0,836 & 0,993 & 0,984 & 1,042 & 0,944 & 1,069 & 0,568 & 1,560 \\
\hline CH Setúbal & 0,930 & 0,811 & 0,930 & 0,995 & 0,999 & 1,049 & 0,940 & 0,958 & 0,710 & 1,574 \\
\hline $\mathrm{CH}$ do Oeste & 1,047 & 1,243 & 0,973 & 1,765 & 1,330 & 0,998 & 1,195 & 0,991 & 1,006 & 1,011 \\
\hline CH de Lisboa Ocidental & 0,921 & 0,806 & 1,089 & 1,073 & 0,913 & 0,928 & 0,898 & 1,034 & 0,635 & 1,388 \\
\hline CH de Lisboa Central & 0,987 & 0,971 & 0,970 & 1,084 & 0,836 & 0,941 & 0,992 & 1,000 & 1,019 & 1,199 \\
\hline CH Lisboa Norte & 0,847 & 0,715 & 1,332 & 1,188 & 0,979 & 0,785 & 0,993 & 1,077 & 0,708 & 1,283 \\
\hline CH do Baixo Alentejo & 1,084 & 1,038 & 0,965 & 1,088 & 0,922 & - & - & - & - & - \\
\hline Média CH & 1,015 & 0,958 & 0,993 & 1,085 & 0,995 & 0,998 & 0,971 & 1,012 & 0,780 & 1,260 \\
\hline
\end{tabular}

Fonte: Elaboração própria. 
Tabela 2. Produtividade média dos Centros Hospitalares antes e após fusão

\begin{tabular}{|c|c|c|c|}
\hline \multirow[b]{2}{*}{$\mathrm{CH}$} & \multicolumn{3}{|c|}{ Indíce de Malquist } \\
\hline & $\begin{array}{l}\text { Antes da } \\
\text { fusão }\end{array}$ & Após fusão & $\Delta \mathrm{I} . \mathrm{Malm}$ \\
\hline CH de São João & 0,979 & 1,008 & $2,90 \%$ \\
\hline $\mathrm{CH}$ Alto Ave & 1,116 & 0,987 & $-12,86 \%$ \\
\hline CH Médio Ave & 1,059 & 0,979 & $-7,97 \%$ \\
\hline $\mathrm{CH}$ entre Douro e Vouga & 0,979 & 0,990 & $1,05 \%$ \\
\hline CH Tâmega e Sousa & 1,037 & 1,014 & $-2,27 \%$ \\
\hline $\begin{array}{l}\text { CH Trás-os-Montes } \\
\text { e Alto Douro }\end{array}$ & 0,981 & 1,040 & $5,87 \%$ \\
\hline $\mathrm{CH}$ do Nordeste & 0,963 & 1,007 & $4,43 \%$ \\
\hline $\mathrm{CH}$ do Porto & 0,999 & 0,933 & $-6,60 \%$ \\
\hline CH V.N.Gaia/Espinho & 0,911 & 0,883 & $-2,80 \%$ \\
\hline CH Leiria-Pombal & 1,119 & 0,890 & $-22,89 \%$ \\
\hline CH Tondela-Viseu & 0,997 & 0,979 & $-1,83 \%$ \\
\hline CH do Baixo Vouga & 0,995 & 0,882 & $-11,38 \%$ \\
\hline $\begin{array}{l}\text { CH Universitário } \\
\text { de Coimbra }\end{array}$ & 1,002 & 1,101 & $9,89 \%$ \\
\hline CH Barreiro - Montijo & 0,972 & 0,973 & $0,07 \%$ \\
\hline CH Setúbal & 0,889 & 1,007 & $11,79 \%$ \\
\hline $\mathrm{CH}$ do Oeste & 1,151 & 1,011 & $-13,92 \%$ \\
\hline CH de Lisboa Ocidental & 0,932 & 0,959 & $2,70 \%$ \\
\hline CH de Lisboa Central & 1,002 & 0,992 & $-0,99 \%$ \\
\hline CH Lisboa Norte & 0,988 & 0,947 & $-4,05 \%$ \\
\hline CH do Baixo Alentejo & 1,061 & 0,989 & $-7,16 \%$ \\
\hline Média CH & 1,004 & 0,977 & $-2,73 \%$ \\
\hline
\end{tabular}

Fonte: Elaboração própria.

exemplos os Centros Hospitalares de Setúbal $(+11,79 \%)$, de Coimbra (+9,89\%), de Trás-os-Montes e Alto Douro (+5,87\%), do Nordeste $(+4,43 \%)$, de São João $(+2,90 \%)$, de Lisboa Ocidental $(+2,70 \%)$, de entre Douro e Vouga $(+1,05 \%)$ e do Barreiro - Montijo (+0,07\%).

Numa perspectiva oposta foram 12 os casos de unidades de saúde que pioraram a sua produtividade com a implementação da política de fusão, a referir: os Centros Hospitalares de Leiria-Pombal (-22,89\%); do Oeste (-13,92\%); do Alto Ave (-12,86\%); de Setúbal (-11,79\%); do Baixo Vouga (-11,38\%); do Médio Ave (-7,97\%); do Baixo Alentejo (-7,16\%), do Porto (-6,60\%); de Lisboa Norte (-4,05\%); de Vila Nova de Gaia/Espinho (-2,80\%); do Tâmega e Sousa (-2,27\%) e de Tondela-Viseu (-1,83\%).

Para poder comparar a evolução da produtividade dos Centros Hospitalares com os hospitais que não foram sujeitos a fusão, efetuou-se com recurso ao índice de Malmquist o apuramento da eficiência para cada um dos cinco hospitais selecionados (Tabela 3).

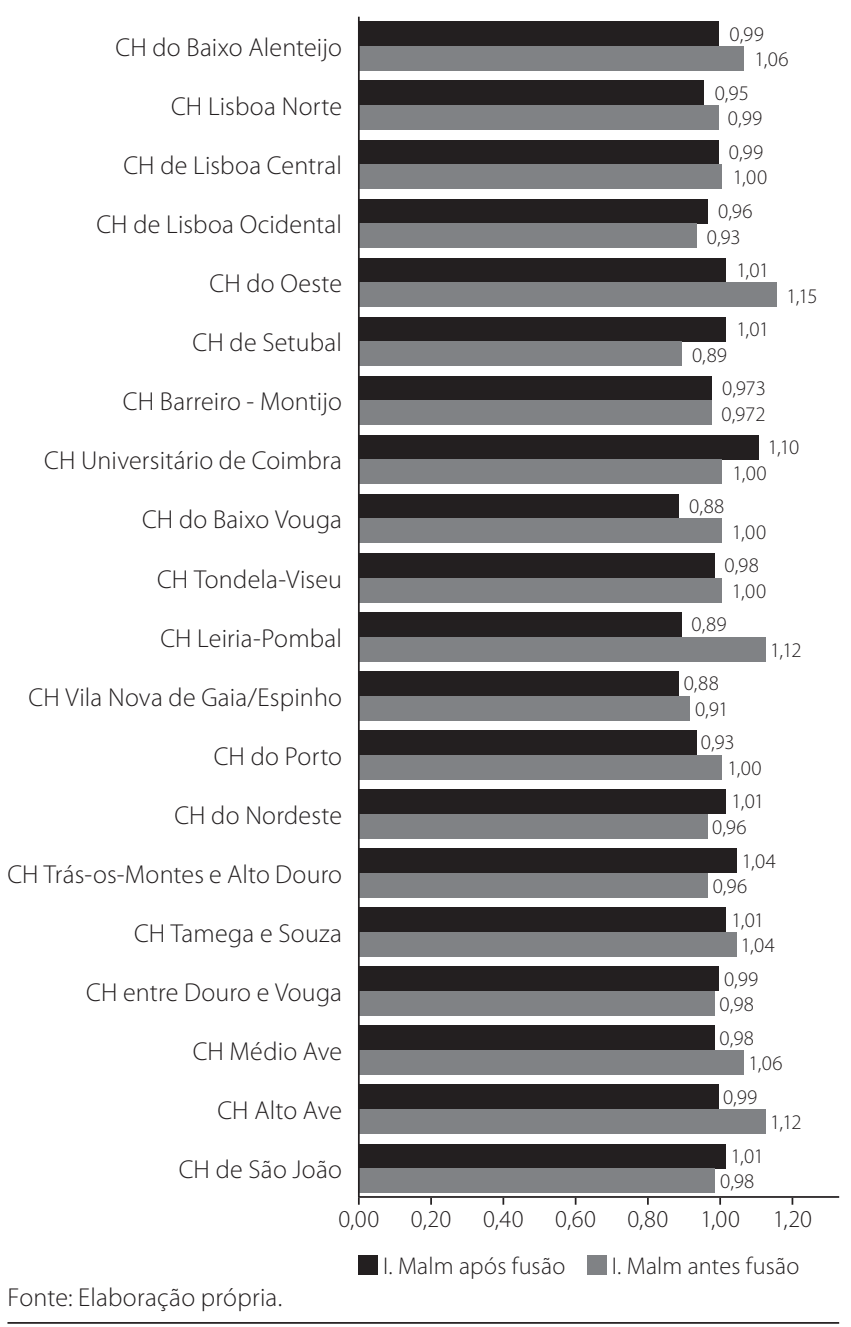

Figura 1. Evolução da produtividade por centro hospitalar, antes e após fusão

A produtividade dos hospitais que não sofreram agregação com outras unidades teve um crescimento médio da sua produtividade em 0,6\%, passando de um score de 1,007 para 1,312 e atravessou um período complicado, tal como os Centros Hospitalares, entre os anos 2010 e 2011 e o impulso de crescimento no ano seguinte. Os fatores justificativos destas variações são os mesmos que foram apontados na análise da variação dos centros hospitalares.

Observando uma análise individual dos hospitais não sujeitos a fusão, apenas o Hospital Distrital da Figueira da Foz e o Hospital Distrital Santa Maria Maior apresentaram produtividades médias positivas com $+0,8 \%$ e $+2,9 \%$, respetivamente.

Quando se considerou a comparação da produtividade do grupo dos Centros Hospitalares criados com os hospitais que não foram sujeitos, obteve-se os seguintes resultados (Figura 2).

A produtividade nos Centros Hospitalares foi superior à dos hospitais nos anos 2003, 2004, 2007, 2010 e 2011. Nos res- 
Tabela 3. Evolução da produtividade dos hospitais não sujeitos a fusões, de 2002 a 2012

\begin{tabular}{lccccccccccc}
\hline Hospital & $\mathbf{2 0 0 3 / 2}$ & $\mathbf{2 0 0 4 / 3}$ & $\mathbf{2 0 0 5 / 4}$ & $\mathbf{2 0 0 6 / 5}$ & $\mathbf{2 0 0 7 / 6}$ & $\mathbf{2 0 0 8 / 7}$ & $\mathbf{2 0 0 9 / 8}$ & $\mathbf{2 0 1 0 / 9}$ & $\mathbf{2 0 1 1 / 1 0}$ & $\mathbf{2 0 1 2 / 1 1}$ & $\begin{array}{c}\text { Média } \\
\mathbf{2 0 1 2 / 0 2}\end{array}$ \\
\hline H. da Figueira da Foz & 1,026 & 0,933 & 1,097 & 1,321 & 0,988 & 0,885 & 0,997 & 0,742 & 0,710 & 1,698 & 1,008 \\
\hline H. de Santarém & 1,080 & 1,002 & 1,004 & 1,019 & 0,961 & 1,053 & 0,960 & 0,909 & 0,566 & 1,661 & 0,991 \\
\hline H. de Santa Maria Maior & 0,987 & 1,207 & 1,071 & 1,109 & 0,965 & 0,966 & 1,098 & 0,939 & 0,966 & 1,012 & 1,029 \\
\hline H. do Espirito Santo & 1,136 & 0,939 & 1,004 & 1,029 & 0,985 & 1,042 & 0,979 & 0,969 & 0,733 & 0,876 & 0,964 \\
\hline Hospital Garcia da Orta & 0,831 & 0,746 & 0,905 & 0,981 & 0,986 & 1,607 & 0,940 & 1,083 & 0,581 & 1,554 & 0,978 \\
\hline Média Hospitais & 1,007 & 0,954 & 1,014 & 1,086 & 0,977 & 1,086 & 0,993 & 0,921 & 0,698 & 1,312 & 0,994 \\
\hline
\end{tabular}

Fonte: Elaboração própria.

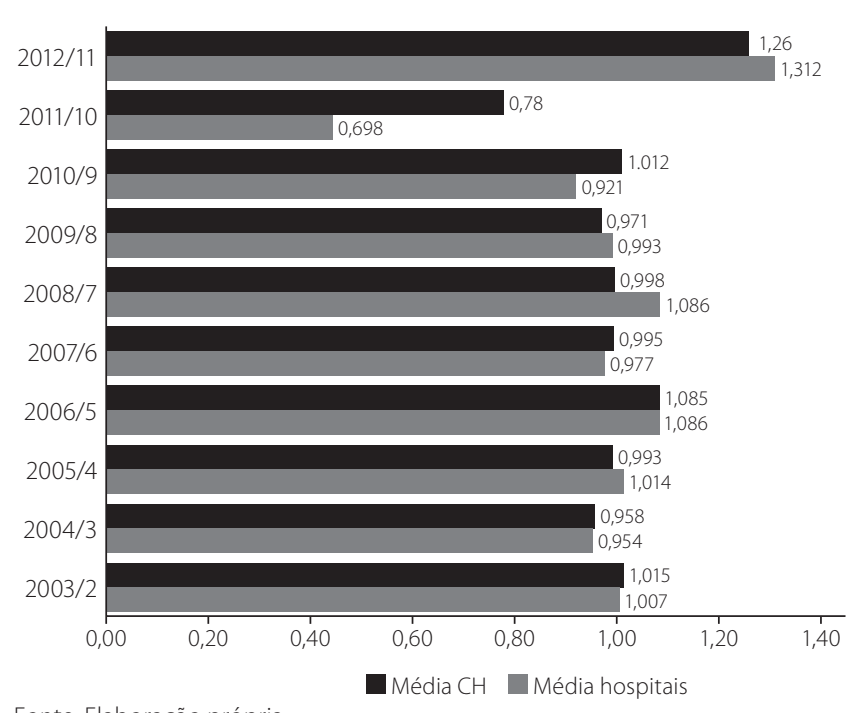

Fonte: Elaboração própria.

Figura 2. Evolução da produtividade por centro hospitalar, antes e após fusão

tantes anos os hospitais não sujeitos a fusão apresentaram um maior desempenho médio. Estes resultados estão equilibrados, pelo que não se poderão obter resultados precisos sobre esta variação.

\section{Discussão}

A implementação das práticas de administração empresarial características do setor privado tinha como objetivos promover: a maior eficiência, o maior acesso e qualidade para o doente.

Além das novas práticas de gestão, os hospitais de média e pequena dimensão foram sujeitos a uma politica de fusão, que tinha como objetivo aproveitar os recursos subaproveitados e assim aumentar a produtividade dos hospitais, formando assim os Centros Hospitalares (Nunes, 2016).

Este processo não foi exclusivo em Portugal e foi implementado em outros países tendo sido obtidos resultados muito positivos. No entanto, nas várias experiências, este modelo de gestão hospitalar teve alguns problemas internos de organização que condicionaram o seu sucesso, pois os hospitais fundiram-se, mas os serviços não. No mesmo Centro Hospitalar continuavam a realizar-se os mesmos serviços em diferentes locais (Lynk, 1995; Tenn, 2011).

Os resultados esperados em termos do aumento de eficiência com as fusões, em Portugal, nos Estados Unidos e no resto do Mundo, só terão efeito nos casos em que se verificasse uma integração das atividades administrativas e clínicas, ficando ambas as unidades a funcionar como uma única unidade (Dranove, 1998; Tenn, 2011).

Neste artigo, os índices de produtividade obtidos foram analisados, em termos médios, para os centros hospitalares (de forma global) e para cada unidade, considerando-se os períodos antes e após fusão. Assim, como principais conclusões se verificou que a produtividade apenas aumentou em $40 \%$ dos Centros Hospitalares e quando comparados com os hospitais não sujeitos a fusão que apresentaram em termos médios uma ligeira melhoria da produtividade no período do estudo.

A crise financeira e a implementação de um programa do ajustamento do FMI poderão ter condicionado os resultados na medida em que a redução da produtividade no ano de 2011 e o significativo aumento no ano seguinte, o que poderá estar associado ao corte no financiamento imposto às unidades hospitalares das unidades, conforme previsto no memorando de entendimento, e não a melhores resultados na produção.

\section{Conclusão}

A política de fusão de unidades de saúde não gerou ganhos de produtividade no médio prazo, sendo, em média, mais produtivos os hospitais que ficaram de fora desse processo.

\section{Referências bibliográficas}

Azevedo H, Mateus C. Economias de escala e de diversificação: uma análise da bibliografia no contexto das fusões hospitalares. Revista Portuguesa de Saúde Pública. 2013;32:106-17.

Barros PP, ed. Economia da saúde - conceitos e comportamentos. 3a edição. Coimbra: Almedina; 2013. 
Brooks GR, Jones VG. Hospital mergers and marker overlap. Health Services resource. 1997;31:701-22.

Campos AC, Simões JM, ed. O percurso da saúde: Portugal na Europa. Coimbra: Almedina; 2011.

Campos AC, ed. Reformas da saúde - o fio condutor. Coimbra: Almedina; 2008.

Campos AC. Por um sistema de saúde mais eficiente. Portugal. Cadernos de economia. 2007;80:9-15.

Caves DW, Christensen LR, Diewert WD. The economic theory of index numbers and the measurement of input, output and productivity. Econometrica. 1982:50:1393-414.

Cooper WW, Seiford LM, Tone K, eds. Data envelopment analysis: a comprehensive text with models, applications, references and deasolver software, 2nd Edition. Reino Unido: Springer; 2007.

Dafny, LS. Estimation and Identification of Merger Effects: An Application to Hospital Mergers. The Journal of Law and Economics. 2009;52:523-50.

Dranove D. Economies of scale in non-revenue producing cost centers: Implications for hospital mergers. Journal of Health Economics. 1998;17:69-83

Ferrier GD, Valdmanis V. (2004). Do mergers improve hospital productivity? Journal of the Operational Research Society. 2004;55;1071-80.

Given RS. Economies of scale and scope as an explanation of merger and output diversification activities in the health maintenance organization industry. Journal of Health Economics. 1996;15;685-713.

Harfouche AP, ed. Opções políticas em saúde. Efeitos sobre a eficiência hospitalar. Coimbra: Almedina; 2012.
Fernandes AC, Nunes AM. Hospitals and the Public-Private Combination in the Portuguese Health System. Acta Médica Portuguesa. 2016;29:217-23.

Lovell CA. Malmquist Productivity Index - Government funded education services. In: Avkiran, NK, ed. Productivity Analysis in the Service Sector with Data Envelopment Analysis. Australia: Universidade de Queensland; 2007. p. 149-61.

Lynk WJ. The creation of economic efficiencies in hospital mergers. Journal of Health Economics. 1995;14:507-30.

Nunes AM, Harfouche AP. A Reforma da Administração Pública aplicada ao setor da saúde: a experiência portuguesa. Brasil. Revista de Gestão em Sistemas de Saúde. 2015:4:1-8.

Nunes AM, ed. Reformas na gestão hospitalar: análise dos efeitos da empresarialização. Lisboa: Instituto Superior de Ciências Sociais e Políticas da Universidade de Lisboa; 2016.

Santana R, Costa C. A integração vertical de cuidados de saúde: aspetos conceptuais e organizacionais. Revista Portuguesa de Saúde Pública. 2008;7:29-56.

Sinay UT. Pre and post-merger investigation of hospital mergers. Eastern Economic Journal. 1998;24:83-97.

Tenn S. The price effects of hospital mergers: a case study of the suttersummit transaction. International Journal of the Economics of Business. 2011;18:65-82.

Vasquez MC, ed. Eficiência e produtividade no ensino superior público. Lisboa: Instituto Superior de Ciências Sociais e Políticas da Universidade de Lisboa; 2012. 